Since the discriminant of the cubic is a square, $b$ can be chosen so that $\alpha^{\prime}=1$. The corresponding value of $\beta^{\prime}$ is

$$
\beta^{\prime}=\frac{-729 \beta^{4}}{\alpha^{3} b^{3}\left(4 \alpha^{3}-27 \beta^{2}\right)} .
$$

The ratio of $\beta^{\prime}$ to $\alpha^{\prime}$ is $-9 \beta^{2} /\left(\alpha^{3} b\right)$. If we take $\alpha$ in (3) to be 1 and determine $b$ in (20) so that $\alpha^{\prime}$ is 1 , we have

$$
\beta^{\prime}=-\beta\left(4-27 \beta^{2}\right)^{1 / 2} \text {. }
$$

We have therefore the following theorem.

THeOREM 3. If $p$ is of the form $6 k-1$ and $x^{3}-x+\beta \equiv 0$ is irreducible, then $x^{3}-x+\beta\left(4-27 \beta^{2}\right)^{1 / 2} \equiv 0$ is also irreducible.

If $\beta$ in the above theorem is not $1 / 3$, the second cubic is distinct from the first. By repeated applications of the theorem we obtain a set of cubics of the form $x^{3}-x+\beta \equiv 0$, but in general we do not obtain all of them.

The University of Illinois

\title{
THE ALGEBRA OF SELF-ADJOINT BOUNDARY-VALUE PROBLEMS*
}

BY V. V. LATSHAW

1. Introduction. By algebraic processes, D. Jackson $\dagger$ obtained in matrix form the condition for self-adjointness of differential systems of any order. The purpose of this paper is to develop by means of the matrix criterion the explicit conditions for selfadjointness of the boundary conditions associated with selfadjoint and anti-self-adjoint differential equations.

2. Even-Order Systems. Let $L(u)$ denote the self-adjoint differential expression $\ddagger$

$$
L(u) \equiv\left(p_{m} u^{(m)}\right)^{(m)}+\left(p_{m-1} u^{(m-1)}\right)^{(m-1)}+\cdots+p_{0} u,
$$

where $m$ is any positive integer, $p_{i}(x)$ is of class $C^{i}$, and $p_{m}(x) \neq 0$ in the interval $(a \leqq x \leqq b)$. Along with

* Presented to the Society, October 31, 1931.

$\dagger$ D. Jackson, Transactions of this Society, vol. 17 (1916), pp. 418-424.

$\ddagger$ Bounitzky, Journal de Mathématiques, (6), vol. 5 (1909), p. 107. 


$$
L(u)=0
$$

is given a set of $2 m$ linearly independent boundary conditions :

$$
U_{k}(u)=\sum_{i=1}^{2 m}\left\{a_{k i} u^{(i-1)}(a)+b_{k i} u^{(i-1)}(b)\right\}=0 .
$$

The theorem of Jackson is as follows.

If the differential expression $L(u)$ is self-adjoint, the condition that the boundary conditions be self-adjoint is that the matrix*

$$
\boldsymbol{\delta}^{\prime} \boldsymbol{\pi}^{(1)} \boldsymbol{\delta}-2 \boldsymbol{\pi}^{(4)} \boldsymbol{\delta}+\boldsymbol{\pi}^{(2)}
$$

be symmetric; if $L(u)$ is an anti-self-adjoint expression or differs from such an expression only in the terms of order zero, the condition is that the matrix just written down be skew symmetric.

If we denote the elements of the non-singular matrix $A_{1}$ by $q_{i j}$ and those of $A_{2}$ by $\dot{q}_{i j}$, we have

$$
A_{1}^{-1}=(Q 1,1 ; 2 m, 2 m)^{\prime} / \bar{A}_{1},
$$

where $Q_{i j}$ represents the cofactor of $q_{i j}$ in $\bar{A}_{1}$. Since $\pi^{(1)}$ is skew symmetric, we find that the element in the $i$ th row and $j$ th column of the matrix $\boldsymbol{\delta}^{\prime} \boldsymbol{\pi}^{(1)} \boldsymbol{\delta}$ may be written

$$
\frac{1}{\bar{A}_{1}^{2}} \sum_{r=1}^{2 m-1} \sum_{s=r+1}^{2 m} \pi_{r s}^{(1)}\left[\sum_{n, k=1}^{2 m} \dot{q}_{n i} \dot{q}_{k j}\left(Q_{n r} Q_{k s}-Q_{n s} Q_{k r}\right)\right] \text {. }
$$

The summation over the indices $n$ and $k$ is equivalent to

$$
\sum_{n=1}^{2 m-1} \sum_{k=n+1}^{2 m}\left[\left(\dot{q}_{n i} \dot{q}_{k j}-\dot{q}_{n j} \dot{q}_{k i}\right)\left(Q_{n r} Q_{k s}-Q_{n s} Q_{k r}\right)\right] .
$$

* We shall adopt the following notation:

$$
(a p, q ; r, s)=\left(\begin{array}{ccc}
a_{p q} & \cdots & a_{p s} \\
\cdots & \cdots & \cdot \\
a_{r q} & \cdots & a_{r s}
\end{array}\right) ; \quad(a p, q ; r, s)^{\prime}=\left(\begin{array}{ccc}
a_{p q} & \cdots & a_{r q} \\
\cdots & \cdots & \cdot \\
a_{p s} & \cdots & a_{r s}
\end{array}\right) .
$$

Boldface letters denote matrices and dashed italics determinants. If we let $A_{1}$ be a square non-singular matrix of the coefficients of $2 m$ variables in $U_{k}(u)$ and $A_{2}$ the matrix of the remaining $2 m$ variables, then $\delta$ denotes the product $A_{1}^{-1} A_{2}$ and $\delta^{\prime}$ the conjugate of $\delta$. Further, we have from Green's formula,

$$
\begin{gathered}
\int_{a}^{b}[v L(u)-u L(v)] d x=\pi(u, v), \\
\pi=\left(\begin{array}{ll}
\boldsymbol{\pi}^{(1)} & \boldsymbol{\pi}^{(3)} \\
\boldsymbol{\pi}^{(4)} & \boldsymbol{\pi}^{(2)}
\end{array}\right)=\left(\begin{array}{l}
\left(\boldsymbol{\pi}^{(1)} 1,1 ; 2 m, 2 m\right)\left(\boldsymbol{\pi}^{(3)} 1,1 ; 2 m, 2 m\right) \\
\left(\boldsymbol{\pi}^{(4)} 1,1 ; 2 m, 2 m\right)\left(\boldsymbol{\pi}^{(2)} 1,1 ; 2 m, 2 m\right)
\end{array}\right) .
\end{gathered}
$$


We replace the quantity $Q_{n r} Q_{k s}-Q_{n s} Q_{k r}$ by $\bar{A}_{1}$ [alg. comp. of $\left.\left(q_{n r} q_{k s}-q_{n s} q_{k r}\right)\right]$ and obtain the further equivalent expression

$$
\begin{array}{r}
\bar{A}_{1}\left[\sum _ { n = 1 } ^ { 2 m - 1 } \sum _ { k = n + 1 } ^ { 2 m } ( \dot { q } _ { n i } \dot { q } _ { k i } - \dot { q } _ { n j } \dot { q } _ { k i } ) \left\{\text { alg. comp. of } \left(q_{n r} q_{k s}\right.\right.\right. \\
\left.\left.\left.-q_{n s} q_{k r}\right)\right\}\right] .
\end{array}
$$

The expansion within the brackets is Laplace's development of the determinant $\bar{A}_{1}$ with the $r$ th and sth columns replaced by the $i$ th and $j$ th columns, respectively, of $A_{2}$. This determinant will be denoted by $D_{r s}(i, j)$.

The application of the criterion of the theorem cited, which required the product of five matrices, is reduced to a process summarized in the following theorem.

THEOREm 1. Given the self-adjoint differential equation, $L(u)$ $=0$, defined by (1), where the coefficients are of class $C^{i}$, and $p_{m}(x)$ $\neq 0$ in the interval $(a \leqq x \leqq b)$, and given a set of $2 m$ linear boundary conditions

$$
U_{k}(u)=\sum_{i=1}^{2 m}\left\{a_{k i} u^{(i-1)}(a)+b_{k i} u^{(i-1)}(b)\right\}=0,
$$

where the determinant $\bar{A}_{1}=\left|q_{i j}\right|$ is of rank $2 m$; then the condition for self-adjointness of the system thus defined, that is, that the matrix

$$
\left(A_{1}^{-1} A_{2}\right)^{\prime} \pi^{(1)} A_{1}^{-1} A_{2}-2 \pi^{(4)} A_{1}^{-1} A_{2}+\pi^{(2)}
$$

be symmetric, reduces by the removal of the factor $A_{1}$ to the following $m(2 m-1)$ conditions:

$$
\begin{aligned}
T_{i j}= & \sum_{r=1}^{2 m-1} \sum_{s=r+1}^{2 m} \pi_{r s}^{(1)} D_{r s}(i, j)-\sum_{r=1}^{2 m} \pi_{i r}^{(4)} D_{r}(j) \\
& +\sum_{r=1}^{2 m} \pi_{j r}^{(4)} D_{r}(i)+\bar{A}_{1} \pi_{i j}^{(2)}=0, \quad(i<j \leqq 2 m) .
\end{aligned}
$$

The symbol $D_{r s}(i, j)$ denotes the determinant $A_{1}$ with the rth and sth columns replaced by the ith and jth columns, respectively, of $\boldsymbol{A}_{2}$; $D_{r}(i)$ indicates one replacement only.

We illustrate the theorem with an example. Let us consider the second-order system 


$$
\begin{aligned}
\left(p_{1} u^{\prime}\right)^{\prime}+p_{0} u=0, \quad \sum_{i=1}^{2}\left\{a_{k i} u^{(i-1)}(a)+b_{k i} u^{(i-1)}(b)\right\} & =0, \\
(k & =1,2) .
\end{aligned}
$$

According to the theorem there is one condition for selfadjointness of the boundary conditions, namely,

$$
\begin{aligned}
T_{12}=\pi_{12}^{(1)} D_{12}(1,2)-\pi_{11}^{(4)} D_{1}(2)-\pi_{12}^{(4)} D_{2}(2)+\pi_{21}^{(4)} D_{1}(1) & \\
& +\pi_{22}^{(4)} D_{2}(1)+\bar{A}_{1} \pi_{12}^{(2)}=0 .
\end{aligned}
$$

For the sake of definiteness let us assume the non-singular matrix to be

$$
A_{1}=\left(\begin{array}{ll}
a_{11} & b_{11} \\
a_{21} & b_{21}
\end{array}\right) ; \quad \text { then } \quad A_{2}=\left(\begin{array}{cc}
a_{12} & b_{12} \\
a_{22} & b_{22}
\end{array}\right) .
$$

Accordingly we have

$$
\begin{aligned}
& \boldsymbol{x}^{(1)}=0, \\
& \pi^{(2)}=0 \text {, } \\
& \boldsymbol{\pi}^{(3)}=\left(\begin{array}{cc}
-p_{1}(a) & 0 \\
0 & p_{1}(b)
\end{array}\right), \quad \boldsymbol{\pi}^{(4)}=\left(\begin{array}{cc}
p_{1}(a) & 0 \\
0 & -p_{1}(b)
\end{array}\right), \\
& T_{12}=p_{1}(a)\left|\begin{array}{ll}
b_{11} & b_{12} \\
b_{21} & b_{22}
\end{array}\right|-p_{1}(b)\left|\begin{array}{ll}
a_{11} & a_{12} \\
a_{21} & a_{22}
\end{array}\right|=0 \text {. }
\end{aligned}
$$

3. Special Cases. In the explicit characterization of specific cases we assume first that in the $2 m$ boundary conditions the sets of terms involving the point $a$ are linearly independent. Under this assumption the condition for self-adjointness becomes

$$
\left(A_{1}^{-1} A_{2}\right)^{\prime} \pi^{(1)} A_{1}^{-1} A_{2}+\pi^{(2)}=0,
$$

subject to the following definitions:

$$
\begin{aligned}
A_{1} & =(a 1,1 ; 2 m, 2 m), \quad A_{2}=(b 1,1 ; 2 m, 2 m), \\
\boldsymbol{\pi} & =\left(\begin{array}{ll}
\boldsymbol{\pi}^{(1)} & \boldsymbol{\pi}^{(3)} \\
\boldsymbol{\pi}^{(4)} & \boldsymbol{\pi}^{(2)}
\end{array}\right)=\left(\begin{array}{cc}
(\alpha 1,1 ; 2 m, 2 m) & 0 \\
0 & (\beta 1,1 ; 2 m, 2 m)
\end{array}\right)^{*} .
\end{aligned}
$$

* The quantities $\alpha_{r s}$ and $\beta_{r s}$ are given by the relations

$$
\alpha_{r s}=(-1)^{r+1} G(a), \quad \beta_{r s}=(-1)^{r} G(b), \text { where } G(x)=\sum_{n}\left(\begin{array}{c}
n-r \\
k
\end{array}\right) p_{n}^{(k)}(x),
$$

if $(r \leqq n \leqq m, s-1), \quad(k=2 n+1-r-s \geqq 0)$, and $r<s$. Further we set

$$
\left(\begin{array}{c}
n-r \\
0
\end{array}\right)=1 \text {. }
$$


We incorporate these results in a corollary.

COROLLARY. If in Theorem 1 we assume that the determinants $\bar{A}_{1}=\left|a_{i j}\right|$ and $\bar{A}_{2}=\left|b_{i j}\right|$ are of rank $2 m$, then the condition for self-adjointness of the system thus defined reduces to the following $m(2 m-1)$ explicit conditions:

$$
T_{i j}=\sum_{r=1}^{m} \sum_{s=r+1}^{2 m} \alpha_{r s} D_{r s}(i, j)+\bar{A}_{1} \beta_{i j}=0, \quad(i<j \leqq 2 m) .
$$

The quantities $\alpha_{r s}$ and $\beta_{r s}$ are computed from the relations (3).

An example will illustrate the corollary. Let us consider the second-order system

$$
\left(p_{1} u^{\prime}\right)^{\prime}+p_{0} u=0, \quad \sum_{i=1}^{2}\left\{a_{k i} u^{(i-1)}(a)+b_{k i} u^{(i-1)}(b)\right\}=0 .
$$

One explicit condition for self-adjointness is obtained:

$$
T_{12}=\alpha_{12} D_{12}(1,2)+\bar{A}_{1} \beta_{12}=0 .
$$

From (3) we have $\alpha_{12}=p_{1}(a)$ and $\beta_{12}=-p_{1}(b)$; also we observe

$$
D_{12}=\left|\begin{array}{ll}
b_{11} & b_{12} \\
b_{21} & b_{22}
\end{array}\right| \text { and } \bar{A}_{1}=\left|\begin{array}{ll}
a_{11} & a_{12} \\
a_{21} & a_{22}
\end{array}\right| \text {. }
$$

We now write the condition in the well known form

$$
T_{12}=p_{1}(a)\left|\begin{array}{ll}
b_{11} & b_{12} \\
b_{21} & b_{22}
\end{array}\right|-p_{1}(b)\left|\begin{array}{ll}
a_{11} & a_{12} \\
a_{21} & a_{22}
\end{array}\right|=0
$$

As a second special case let us consider a system consisting of the self-adjoint differential equation defined in (2) and a set of $2 m$ linearly independent Sturmian boundary conditions

$$
\begin{aligned}
& U_{k}^{(a)}=\sum_{i=1}^{2 m} a_{k i} u^{(i-1)}(a)=0, \quad(k=1,2, \cdots, h), \\
& U_{k}^{(b)}=\sum_{i=1}^{2 m} b_{k i} u^{(i-1)}(b)=0, \quad(k=1,2, \cdots, f) .
\end{aligned}
$$

It can be shown* that if $h$ of the given boundary conditions

* D. Jackson, Proceedings of the American Academy of Arts and Sciences, vol. 51, pp. 403-417. 
involve only the point $a$, then $h$ of the adjoint conditions will involve only the point $b$; consequently for $h \neq f$ self-adjointness is impossible. We shall assume $h=f=m$ and that the sets of terms involving the variables $u(a), u^{\prime}(a), \cdots, u^{(m-1)}(a)$ and likewise those involving the variables $u(b), u^{\prime}(b), \cdots, u^{(m-1)}(b)$ are linearly independent. Following the process previously outlined, we find

$$
\begin{aligned}
A_{1} & =\left(\begin{array}{ll}
a & 0 \\
0 & b
\end{array}\right)=\left(\begin{array}{cc}
(a 1,1 ; m, m) & 0 \\
0 & (b 1,1 ; m, m)
\end{array}\right), \\
A_{2} & =\left(\begin{array}{cc}
(a 1, m+1 ; m, 2 m) & 0 \\
0 & (b 1, m+1 ; m, 2 m)
\end{array}\right) .
\end{aligned}
$$

Further, let $D_{r}(i)$ represent the determinant $\bar{a}$ with elements $a_{k r}$ replaced by elements $a_{k i}$. Also let $D_{r s}(i, j)$ denote $\bar{a}$ with $a_{k r}$ and $a_{k s}$ replaced by $a_{k i}$ and $a_{k j}$ respectively. Similarly, we represent the determinants involving $b$ by $D_{r}^{\prime}(i)$ and $D_{r s}^{\prime}(i, j)$.

After making certain algebraic reductions, we are able to express the product matrices $\boldsymbol{\delta}^{\prime} \boldsymbol{\pi}^{(1)} \boldsymbol{\delta}$ and $\delta \boldsymbol{\pi}^{(4)}$ in concise form:

$$
\begin{aligned}
\boldsymbol{\delta}^{\prime} \boldsymbol{\pi}^{(1)} \boldsymbol{\delta} & =\left(\begin{array}{cc}
\left(a^{\prime} 1,1 ; m, m\right) & 0 \\
0 & \left(b^{\prime} 1,1 ; m, m\right)
\end{array}\right), \\
\boldsymbol{\pi}^{(4)} \boldsymbol{\delta} & =\left(\begin{array}{cc}
\left(a^{\prime \prime} 1,1 ; m, m\right) & 0 \\
0 & \left(b^{\prime \prime} 1,1 ; m, m\right)
\end{array}\right),
\end{aligned}
$$

where

and

$$
\begin{aligned}
& a_{i j}^{\prime}=\frac{1}{\bar{a}} \sum_{r=1}^{m-1} \sum_{s=r+1}^{m} \alpha_{r s} D_{r s}(m+i, m+j), \\
& b_{i j}^{\prime}=\frac{1}{\bar{b}} \sum_{r=1}^{m-1} \sum_{s=r+1}^{m} \beta_{r s} D_{r s}^{\prime}(m+i, m+j),
\end{aligned}
$$

$$
a_{i j}^{\prime \prime}=\frac{1}{\bar{a}} \sum_{s=1}^{m} \alpha_{m+j, 8} D_{s}(m+i), \quad b_{i j}{ }^{\prime \prime}=\frac{1}{\bar{b}} \sum_{s=1}^{m} \beta_{m+i, s} D_{s}^{\prime}(m+i) .
$$

We summarize these results in a theorem.

THEOREM 2. Given the self-adjoint differential equation $L(u)$ $=0$, defined by (1), where the coefficients are of class $C^{i}$ and we assume $p_{m}(x) \neq 0$ in the interval $(a \leqq x \leqq b)$; and given a set of $2 m$ linearly independent Sturmian boundary conditions: 
1933.]

$$
\begin{array}{r}
U_{k}(a)=\sum_{i=1}^{2 m} a_{k i} u^{(i-1)}(a)=0, \quad U_{k}(b)=\sum_{i=1}^{2 m} b_{k i} u^{(i-1)}(b)=0, \\
(k=1,2, \cdots, m),
\end{array}
$$

in which $\left|a_{k s}\right|,\left|b_{k s}\right| \neq 0$ for $s=1,2, \cdots, m$; the condition for self-adjointness of the system thus defined, that is, that the matrix

$$
\left(A_{1}^{-1} A_{2}\right)^{\prime} \pi^{(1)} A_{1}^{-1} A_{2}-2 \pi^{(4)} A_{1}^{-1} A_{2}+\pi^{(2)}
$$

be symmetric, reduces by the removal of common factors to the following $m(m-1) / 2$ conditions at each end point:

$$
\begin{gathered}
T_{i j}(a)=\sum_{r=1}^{m-1} \sum_{s=r+1}^{m} \alpha_{r s} D_{r s}(m+i, m+j)+\sum_{s=1}^{m} \alpha_{m+j, s} D_{s}(m+i) \\
\quad-\sum_{s=1}^{m} \alpha_{m+i, s} D_{s}(m+j)=0, \\
T_{i j}(b)=\sum_{r=1}^{m-1} \sum_{s=r+1}^{m} \beta_{r s} D_{r s}^{\prime}(m+i, m+j)+\sum_{s=1}^{m} \beta_{m+j, s} D_{s}^{\prime}(m+i) \\
\quad-\sum_{s=1}^{m} \beta_{m+i, s} D_{s}^{\prime}(m+j)=0, \quad(i<j \leqq m),
\end{gathered}
$$

in which the abbreviations defined in (3) and (4) are used.

Example 1. $m=1$. According to the requirements of Theorem 2 this system is always self-adjoint. It is of interest to check this result by direct calculation.

$$
\begin{aligned}
\int_{a}^{b}[v L(u)-u L(v)] d x= & {\left[p_{1}(x)\left(v u^{\prime}-u v^{\prime}\right)\right]_{a}^{b} } \\
& =U_{1} V_{4}+U_{2} V_{3}+U_{3} V_{2}+U_{4} V_{1},
\end{aligned}
$$

where $U_{1}=a_{11} u(a)+a_{12} u^{\prime}(a), U_{2}=b_{11} u(b)+b_{12} u^{\prime}(b)$. Further we assume $U_{3}=u^{\prime}(a), U_{4}=u^{\prime}(b)$; then

$$
\begin{aligned}
& V_{1}=p_{1}(b)\left[b_{11} v(b)+b_{12} v^{\prime}(b)\right] / b_{11}, \\
& V_{2}=-p_{1}(a)\left[a_{11} v(a)+a_{12} v^{\prime}(a)\right] / a_{11} .
\end{aligned}
$$

Since $V_{1}$ and $V_{2}$ are essentially $U_{2}$ and $U_{1}$, respectively, the system is self-adjoint. 
EXAmple 2. $m=2$.

$$
\begin{aligned}
T_{12}(a)= & p_{1}(a)\left|\begin{array}{ll}
a_{13} & a_{14} \\
a_{23} & a_{24}
\end{array}\right|-p_{2}(a)\left|\begin{array}{ll}
a_{13} & a_{12} \\
a_{23} & a_{22}
\end{array}\right| \\
& +p_{2}^{\prime}(a)\left|\begin{array}{ll}
a_{14} & a_{12} \\
a_{24} & a_{22}
\end{array}\right|-p_{2}(a)\left|\begin{array}{ll}
a_{11} & a_{14} \\
a_{21} & a_{24}
\end{array}\right|=0 \\
-T_{12}(b)= & p_{1}(b)\left|\begin{array}{ll}
b_{13} & b_{14} \\
b_{23} & b_{24}
\end{array}\right|-p_{2}(b)\left|\begin{array}{ll}
b_{13} & b_{12} \\
b_{23} & b_{22}
\end{array}\right| \\
& +p_{2}^{\prime}(b)\left|\begin{array}{ll}
b_{14} & b_{12} \\
b_{24} & b_{22}
\end{array}\right|-p_{2}(b)\left|\begin{array}{ll}
b_{11} & b_{14} \\
b_{21} & b_{24}
\end{array}\right|=0
\end{aligned}
$$

4. Odd-Order Systems. In general we shall use the same method but some modification is required. Let $L(u)$ represent the anti-self-adjoint differential expression

$$
\begin{aligned}
L(u) \equiv\left(p_{n} u\right)^{(n)}+\left(p_{n-2} u\right)^{(n-2)} & +\cdots+\left(p_{1} u\right)^{\prime}+p_{n} u^{(n)} \\
& +p_{n-2} u^{(n-2)}+\cdots+p_{1} u^{\prime}
\end{aligned}
$$

where $n$ is any positive odd integer, $p_{i}(x)$ is of class $C^{i}$, and $p_{n}(x)$ $\neq 0$ in the interval $(a \leqq x \leqq b)$. Along with

$$
L(u)=0
$$

is given a set of linearly independent boundary conditions

$$
U_{k}=\sum_{i=1}^{n}\left\{a_{k i} u^{(i-1)}(a)+b_{k i} u^{(i-1)}(b)\right\}=0,(k=1,2, \cdots, n) .
$$

In our usual notation the product matrix $\delta^{\prime} \boldsymbol{\pi}^{(1)} \delta$ may be written

$$
\boldsymbol{\delta}^{\prime} \boldsymbol{\pi}^{(1)} \boldsymbol{\delta}=\frac{1}{\bar{A}_{1}^{2}}\left(a^{\prime} 1,1 ; n, n\right), \text { where } a_{i j}{ }^{\prime}=\sum_{r, s=1}^{n} \pi_{r s}^{(1)} D_{r}(i) D_{s}(j) .
$$

Since $\pi^{(1)}$ is not skew symmetric for the odd-order system, the further reduction by removal of a common factor is no longer possible. We state these results more concisely in the following theorem.

THEOREM 3. Given the anti-self-adjoint differential equation $L(u)=0$, defined by (5), where the coefficients $p_{i}(x)$ are of class $C^{i}$, and $p_{n}(x) \neq 0$ in the interval $(a \leqq x \leqq b)$, and given a set of $n$ linear boundary conditions 


$$
U_{k}=\sum_{i=1}^{n}\left\{a_{k i} u^{(i-1)}(a)+b_{k i} u^{(i-1)}(b)\right\}=0, \quad(k=1,2, \cdots, n),
$$

where the determinant $\bar{A}_{1}=\left|q_{i j}\right|$ is of rank $n$; the condition for self-adjointness of the system thus defined, that is, that the matrix

$$
\left(A_{1}^{-1} A_{2}\right)^{\prime} \pi^{(1)} A_{1}^{-1} A_{2}-2 \pi^{(4)} A_{1}^{-1} A_{2}+\pi^{(2)}
$$

be skew symmetric, reduces to the following $n(n+1) / 2$ conditions:

$$
\begin{aligned}
T_{i j}= & \sum_{r, s=1}^{n} \pi_{r s}^{(1)} D_{r}(i) D_{s}(j)-\bar{A}_{1} \sum_{r=1}^{n} \pi_{i r}^{(4)} D_{r}(j) \\
& -\bar{A}_{1} \sum_{r=1}^{n} \pi_{j r}^{(4)} D_{r}(i)+\bar{A}_{1}^{2} \pi_{i j}^{(2)}=0,(i \leqq j \leqq n) .
\end{aligned}
$$

To illustrate the theorem let us consider the first-order system

$$
\left(p_{1} u\right)^{\prime}+p_{1} u^{\prime}=0, \quad a_{11} u(a)+b_{11} u(b)=0 .
$$

Assume $A_{1}=b_{11}$; then we have $A_{2}=a_{11}$, and

$$
\stackrel{(1)}{\pi_{11}} D_{1}^{2}(1)+\stackrel{A_{1}^{2} \pi_{11}^{(2)}}{=}=0=a_{11}^{2} p_{1}(b)-b_{11}^{2} p_{1}(a) .
$$

5. Special Cases. If we assume that in the $n$ boundary conditions the sets of terms involving the point $a$ are linearly independent, the condition for self-adjointness becomes

$$
\left(A_{1}^{-1} A_{2}\right)^{\prime} \pi^{(1)} A_{1}^{-1} A_{2}+\pi^{(2)}=0,
$$

subject to the following definitions:*

$$
\begin{gathered}
A_{1}=(a 1,1 ; n, n), \quad A_{2}=(b 1,1 ; n, n), \\
\boldsymbol{\pi}=\left(\begin{array}{cc}
(\alpha 1,1 ; n, n) & 0 \\
0 & (\beta 1,1 ; n, n)
\end{array}\right)=\left(\begin{array}{cc}
\boldsymbol{\pi}^{(1)} & \boldsymbol{\pi}^{(3)} \\
\boldsymbol{\pi}^{(4)} & \boldsymbol{\pi}^{(2)}
\end{array}\right) .
\end{gathered}
$$

If we let $D_{r}(i)$ denote the determinant $\bar{A}_{1}$ with the elements $a_{k r}$ replaced by the elements $b_{k i}$, the product matrix $\delta^{\prime} \boldsymbol{\pi}^{(1)} \boldsymbol{\delta}$ may be written

$$
\begin{aligned}
& { }^{*} \alpha_{r s}=\alpha_{s r}=\sum_{m} G_{m} p_{2 m+1}^{(k)}(a) ; \quad \beta_{r s}=\beta_{s r}=-\sum_{m} G_{m} p_{2 m+1}^{(k)}(b), \\
& \text { where } \left.\quad \begin{array}{c}
{ }^{(k)} \\
k
\end{array}\right)+(-1)^{r}\left(\begin{array}{c}
2 m-r+1 \\
k
\end{array}\right),
\end{aligned}
$$

if $k=2 m-r-s+2 \geqq 0$ and $0 \leqq m \leqq(n-1) / 2$. 


$$
\boldsymbol{\delta}^{\prime} \boldsymbol{\pi}^{(1)} \boldsymbol{\delta}=\frac{1}{\bar{A}_{1}^{2}}\left(a^{\prime} 1,1 ; n, n\right), \text { where } a_{i j}^{\prime}=\sum_{r, s=1}^{n} \alpha_{r s} D_{r}(i) D_{s}(j) .
$$

These results may be stated in a corollary.

COROLlARY. If in Theorem 3 we assume that the determinants $\bar{A}_{1}=\left|a_{i j}\right|$ and $\bar{A}_{2}=\left|b_{i j}\right|$ are of rank $n$, then the condition for selfadjointness of the system thus defined reduces to the following $n(n+1) / 2$ explicit conditions:

$$
T_{i j}=\sum_{r, s=1}^{n} \alpha_{r s} D_{r}(i) D_{s}(j)+\bar{A}_{1}^{2} \beta_{i j}=0, \quad(i \leqq j \leqq n) .
$$

The quantities $\alpha_{r s}$ and $\beta_{r s}$ are obtained from the relations (6).

An example will suffice to illustrate the corollary. For $n=1$,

$$
\left(p_{1} u\right)^{\prime}+p_{1} u^{\prime}=0, \quad a_{11} u(a)+b_{11} u(b)=0 .
$$

One condition for self-adjointness is specified, namely:

$$
T_{11}=p_{1}(b) a_{11}^{2}-p_{1}(a) b_{11}^{2}=0 .
$$

In verifying this result by direct calculation we have $\pi(u, v)=2 p_{1}(b) v(b) u(b)-2 p_{1}(a) v(a) u(a)=U_{1} V_{2}+U_{2} V_{1}$, where $U_{1}=a_{11} u(a)+b_{11} u(b)$ and we let $U_{2}=u(b)$. We find

$$
V_{1}=2 p_{1}(b) v(b)+2 p_{1}(a) v(a) b_{11} / a_{11} .
$$

Obviously for $V_{1}$ to be essentially $U_{1}$ the condition stated above must hold.

In considering odd-order systems with Sturmian boundary conditions it is sufficient to observe that the conditions pertaining to the end points are unequal in number; consequently self-adjointness of such systems is impossible.

LEHIGH UNIVERSITY 